\title{
High prevalence of norovirus GII.P16/GII.2 and chicken anemia virus in two acute gastroenteritis outbreaks in Huzhou, China
}

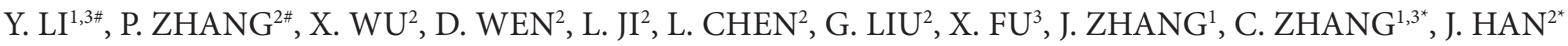

\begin{abstract}
${ }^{1}$ The Joint Center for Infection and Immunity: Guangzhou Institute of Pediatrics, Guangzhou Women and Children's Medical Center, Guangzhou, 510623 and Institut Pasteur of Shanghai, Chinese Academy of Sciences, Shanghai, 200031, P. R. China; ${ }^{2}$ Huzhou Center for Disease Control and Prevention, Huzhou, 313000, P. R. China; ${ }^{3}$ Pathogen Discovery and Big Data Center, CAS Key Laboratory of

Molecular Virology \& Immunology, Institut Pasteur of Shanghai, Chinese Academy of Science, Shanghai, 200031, P. R. China
\end{abstract}

Received September 09, 2018; revised November 11, 2018; accepted July 29, 2019

\begin{abstract}
Summary. - Acute gastroenteritis (AGE) is one of the most frequently occuring illnesses in children and adults worldwide. In February 2017, two AGE outbreaks occurred in two adjacent schools in Huzhou city, Zhenjiang province of China. We detected high percentages of recombinant norovirus GII.P16/GII.2 in one school and chicken anemia virus (CAV) in another school using next generation sequencing (NGS) and specific PCR. The results highlight the importance of continuous surveillance of GII.P16/GII.2, and suggest the need of further studies on whether CAV causes AGE.
\end{abstract}

Keywords: acute gastroenteritis; norovirus; chicken anemia virus; Huzhou; School

Acute gastroenteritis (AGE) is one of the most frequently occuring illnesses in children and adults worldwide, and most AGE can be explained by a viral etiology. More than 20 different types of viruses are known to be associated with AGE, of which norovirus is the most frequently detected virus (Patel et al., 2009). Noroviruses can be classified into seven genogroups (GI-GVII) according to the characteristics of the RdRp and VP1 gene sequences. A recombinant norovirus, GII.P16-GII.2 surpassed previously predominant GII. 4 as a predominant GII genotype and was responsible for majority of the AGE outbreaks in China since 2016 (Ao et al., 2017; Lu et al., 2017). Chicken anemia virus (CAV) belongs to the Gyrovirus genus in the Circoviridae family, and was often detected in stool samples of animals and humans. Up to now, there is no direct evidence to support the association of CAV with human AGE. In this study, we reported two AGE outbreaks in two adjacent schools and

*Corresponding authors. E-mail: hanjk678@163.com (J. Han) or zhangcy1999@ips.ac.cn (C. Zhang); phone: +86-572-2760 112. \#These authors contributed equally to this study.

Abbreviations: $\mathrm{AGE}=$ acute gastroenteritis; $\mathrm{CAV}(\mathrm{s})=$ chicken anemia virus(es) identified norovirus GII.P16/GII.2 in one school and CAV in another school.

In February 2017, two AGE outbreaks occurred in two adjacent schools in Huzhou city, Zhejiang province of China (Fig. 1a). The AGE outbreak occurring in School A lasted from Feb. 13 to Mar. 6, 2017, with a peak on Feb. 15 and Feb. 16 (Fig. 1b). The outbreak in School B started on Feb.16, when the epidemic peak occurred in the School A, suggesting a potential epidemiological link between the AGE outbreaks in the two schools. The outbreak in the School B peaked in on Feb. 27 and Feb. 28, and stopped on Mar. 1 (Fig. 1b).

Vomiting, diarrhea and abdominal pain were the main symptoms presented during the two AGE outbreaks. In the School A, 60 (56.1\%), 80 (75.7\%) and 90 (84.1\%) patients displayed vomiting, diarrhea and abdominal pain, respectively, while in the school B the numbers were $22(26.5 \%)$, $74(89.2 \%)$ and 48 (57.8\%), respectively. The proportions of vomiting and abdominal pain were substantially higher in the School A than in the School B, possibly implying more severe clinical manifestations in the School A. The difference in symptoms between the two AGE outbreaks might imply that the AGE outbreaks were caused by different pathogens (Table 1). 
(a)
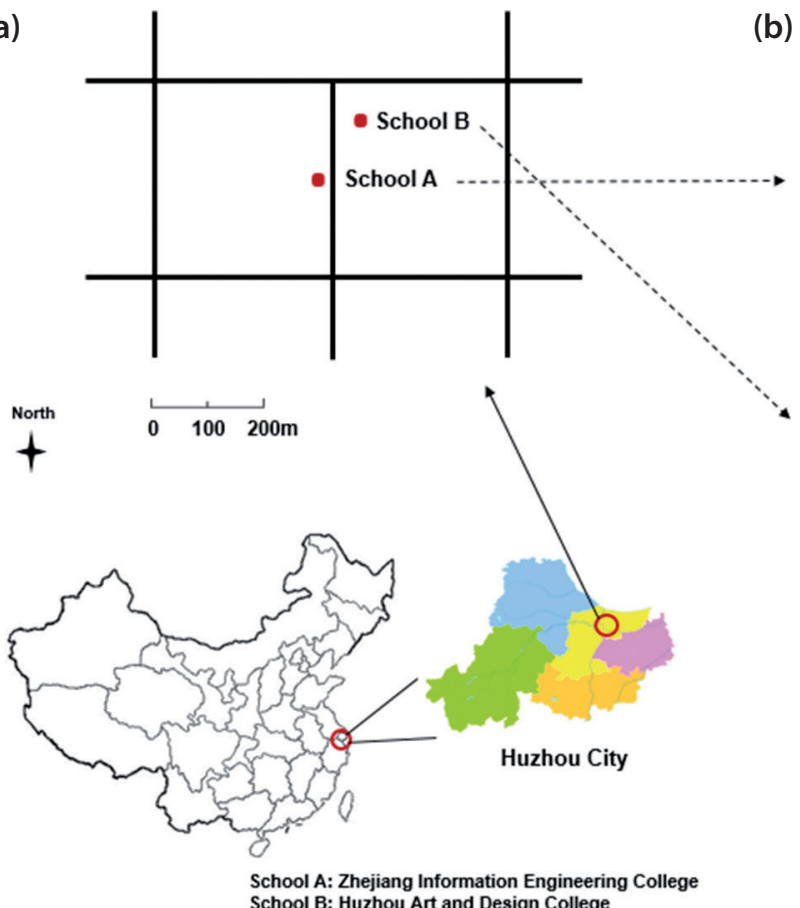

(b)

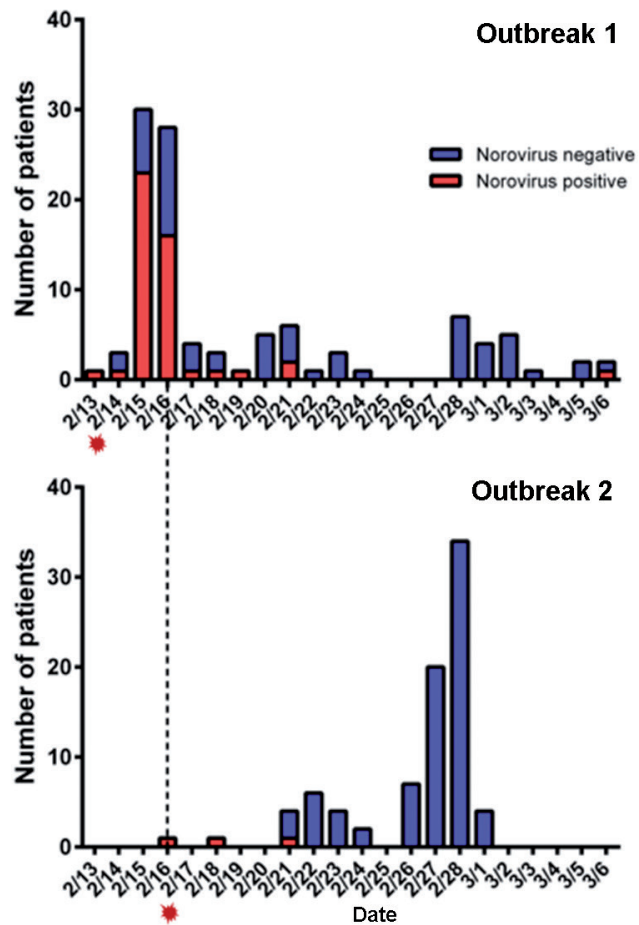

Fig. 1

Characteristics and daily records of the two AGE outbreaks

(a) location of the two AGE outbreaks. (b) the Characteristics and daily records.

Table 1. Clinical characteristics of AGE patients and detection of viruses in the two schools

\begin{tabular}{|c|c|c|c|}
\hline & School A & School B & $P$-value \\
\hline Case number & 107 & 83 & - \\
\hline Male / Female & $95 / 12$ & $13 / 70$ & $P<0.001$ \\
\hline Norovirus-positive & $47(43.9 \%)$ & $3(3.6 \%)$ & $P<0.001$ \\
\hline CAV-positive & $12.1 \%(13 / 107)$ & $35 \%(28 / 80)$ & $P<0.001$ \\
\hline Vomiting & $60(56.1 \%)$ & $22(26.5 \%)$ & $P<0.001$ \\
\hline Diarrhea & $81(75.7 \%)$ & $74(89.2 \%)$ & $P=0.023$ \\
\hline Both vomiting and diarrhea & $39(36.4 \%)$ & $15(18.1 \%)$ & $P=0.006$ \\
\hline Abdominal pain & $90(84.1 \%)$ & $48(57.8 \%)$ & $P<0.001$ \\
\hline Fever & $12(11.2 \%)$ & $6(7.2 \%)$ & $P=0.456$ \\
\hline
\end{tabular}

Notes: Fisher exact test was used for the comparison between the two schools.

To determine the causative agents for the two outbreaks, the vomiting or stool samples were collected from each patient and tested for noroviruses using a commercial real-time reverse transcription PCR kit (Zhijiang Bio-Tech, Shanghai). In the School A, 43.9\% (47/107) of the samples were positive for norovirus, significantly higher than $3.6 \%(3 / 83)$ in the School B $(P<0.001)$ (Table 1$)$. The extremely low positive rate $(3.6 \%)$ of norovirus in the School B excluded the epide- miological link between the two schools and suggested that there might be other pathogens responsible for the AGE.

To determine the genotype of the norovirus in the School A, 9 randomly selected samples were subjected to sequencing and phylogenetic analyses. The 3 ' end of the polymerase gene (region A in ORF1) and the 5 ' end of the capsid gene (region C in ORF2) of norovirus were amplified and sequenced using primers JV12Y/JV13I and G2SKF/ 

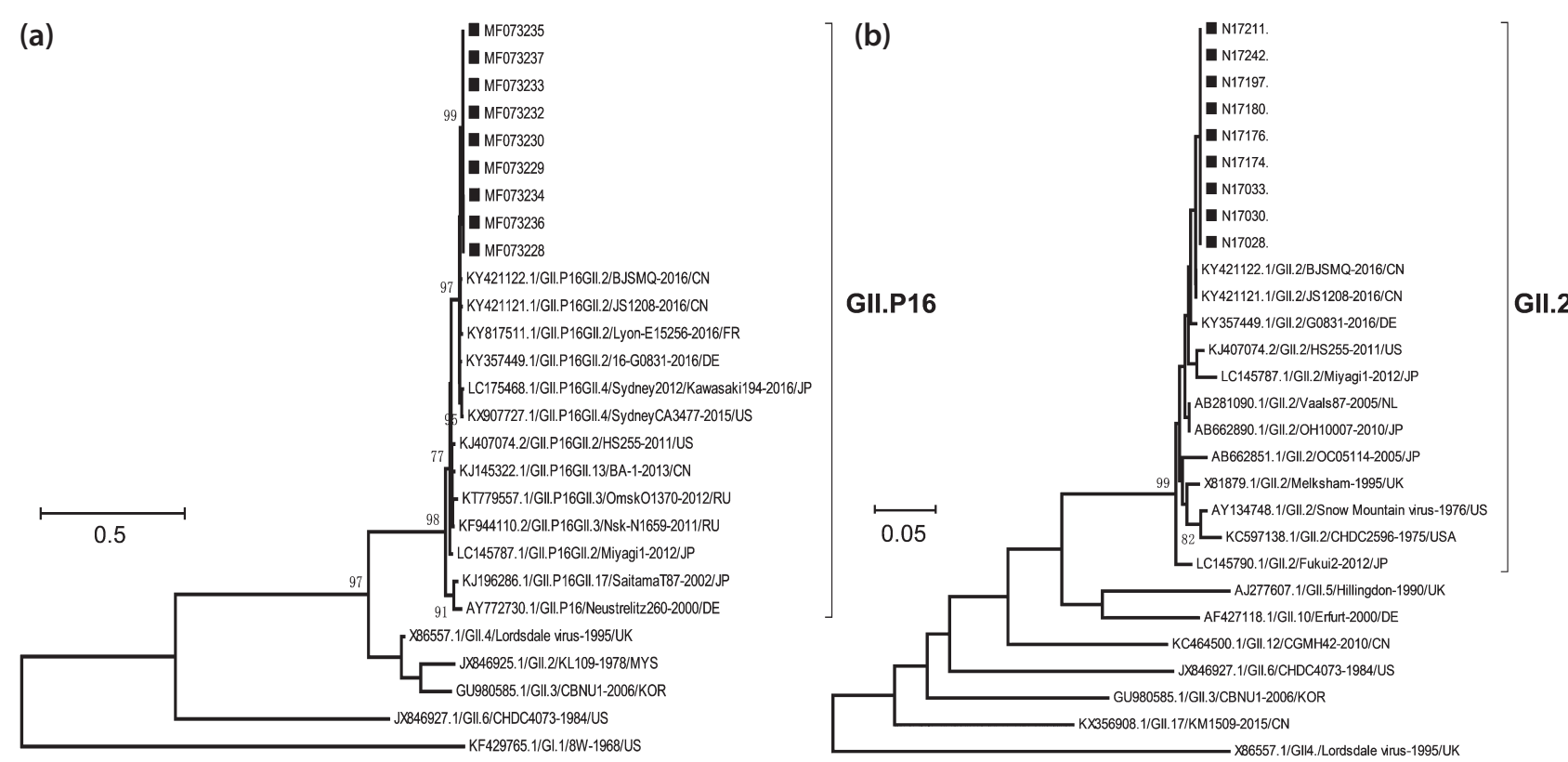

Fig. 2

Phylogenetic analyses of the norovirus identified in the School A The neighbor-joining (NJ) tree of partial RNA-dependent RNA polymerase (a) partial capsid genes (b) were constructed using MEGA 7.0. The sequences obtained in this study are highlighted by solid squares.

G2SKR described previously (Kojima et al., 2002; Vinje et al., 2003). Phylogenetic analyses of partial capsid genes and $R d R p$ genes showed that all sequences from the 9 samples closely clustered with the GII.P16/GII.2 norovirus sequences from the 2016 outbreaks in China and Germany (Ao et al., 2017; Lu et al., 2017) (Fig. 2). The 9 GII.P16/GII.2 sequences showed $99 \%$ identity with each other. These results indicated that the AGE outbreak in the School A was caused by the recombinant norovirus GII.P16/GII.2.

To identify the causative agent responsible for the AGE outbreak in School B, we first used a commercial PCR panel (Zhijiang Bio-Tech, Shanghai) to detect common viruses that can cause gastroenteritis, including enterovirus, asteroid virus, rotavirus, adenovirus and sapovirus. All norovirus-negative samples from the school B were also negative for these tested viruses. Viral metagenomic analysis is an unbiased approach for pathogen identification and discovery (Li et al., 2018; Wang et al., 2019). To further investigate the reason of the AGE outbreak in the School B, we analyzed 4 norovirus-negative samples from the School B, as well as two norovirus-negative and two norovirus-positive samples from the School A using the viral metagenomic analysis. The sample processing and enrichment of encapsidated DNA and RNA viruses were performed using a viral metagenomics pipeline described in detail by Legoff et al. (2017). A random-amplification approach (REPLI-g Single Cell WTA kit, Qiagen, Germany) was used to amplify the total nucleic acids (DNA and RNA) from the samples. Illumina HiSeq 2000 platform was used for deep sequencing. The metagenomic analyses confirmed the presence of norovirus in the two norovirus-positive samples from the School A, and the absence of norovirus, enterovirus, asteroid virus, rotavirus, adenovirus and sapovirus in other six norovirus-negative samples from both Schools A and B.

Importantly, we found very high abundance $(50 \%-98 \%)$ of CAV reads in three norovirus-negative samples from the School B (Fig. 3a). Other viruses, including parvovirus, endogenous retrovirus, viruses of the family Genomoviridae and plant viruses were also detected with very low proportions. The presence of $\mathrm{CAV}$ in the three samples was confirmed by a specific PCR assay. We then detected the presence of CAV in other stool and vomiting samples from both schools, and found that $12.1 \%$ (13/107) of samples in the School A and 35.0\% (28/80) of samples in the School B were positive (Table 1 ). The positive rate of CAV was significantly higher in the School B than in the School A $(P<0.001)$. These results suggest a potential association of $C A V$ with the AGE. We further sequenced the CAV from the samples, and performed a phylogenetic analysis. The CAV sequences from both schools often clustered together but separately from the reference sequences from other countries or regions (Fig. 3b). This result suggests that the CAV strains from both schools were genetically related. 


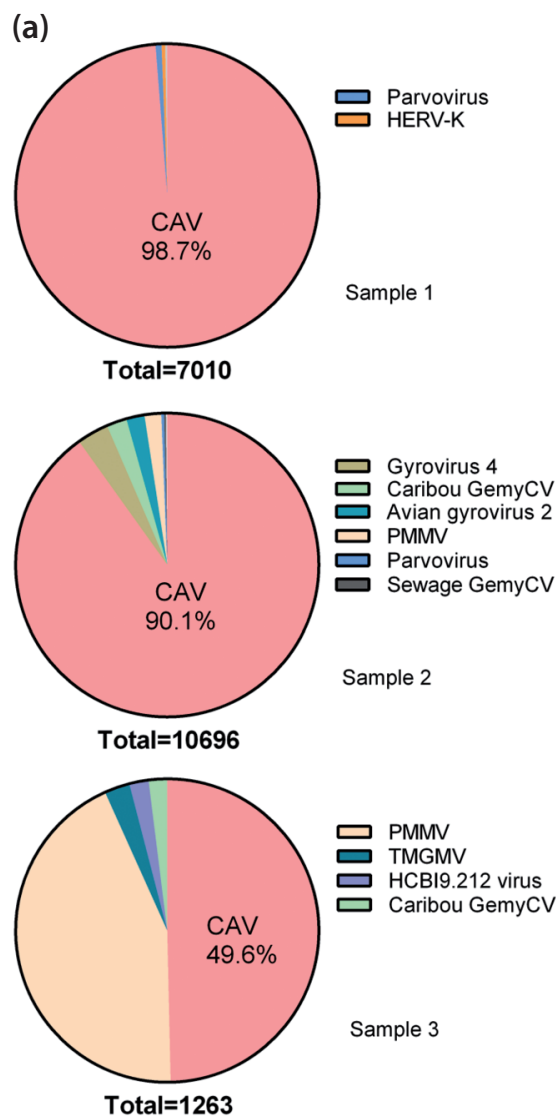

Viral metagenomic sequencing

(b)

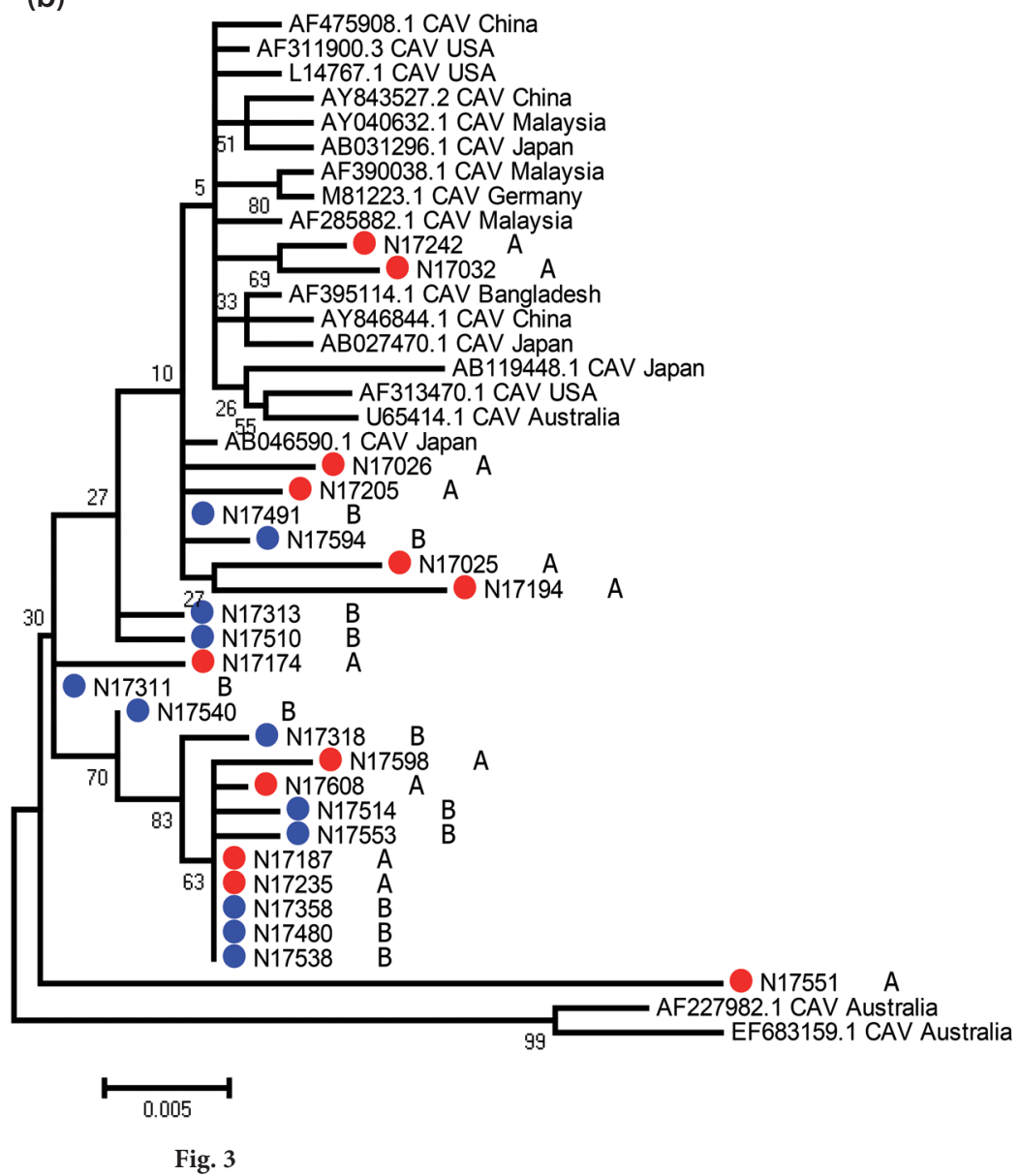

Detection of chicken anemia virus

(a) NGS reads distribution of the three norovirus-negative samples from the School B. Only viruses that had more than 15 reads are shown. (b) Phylogenetic analyses of the CAV sequences obtained from the Schools A (red solid circle) and B (blue solid circle) with reference strains from other countries or regions. The NJ tree were constructed based on partial VP1 gene ( 900bp) of CAV using MEGA 7.0.

Norovirus and some other viruses (e.g. enterovirus, asteroid virus, rotavirus, adenovirus and sapovirus) are the main cause of non-bacterial AGE. The AGE caused by different viruses often exhibits various clinical symptoms. In this study, obviously different symptom profiles of AGE outbreaks were observed in the two schools (Table 1), suggesting different causative agents causing the two AGE outbreaks. By specific RT-qPCR assays, we identified the recombinant norovirus GII.P16/GII.2 as the main cause of the AGE outbreak in the School A, but failed to detect any above-mentioned viruses in the School B except three norovirus-positive cases, implying the presence of a different causative agent. Using the next generation sequencing, we found extremely high abundance of CAV reads in three samples from the School $B$, and detected a higher prevalence of $\operatorname{CAV}(35 \%, 28 / 80)$ in the School B than in the School A $(12.1 \%, 13 / 107)$ (Table 1), suggesting a potential association of CAV with AGE.
CAV belongs to the genus Gyrovirus (the family Circoviridae) and contains a circular single-stranded DNA genome of about $2.3 \mathrm{~kb}$ (Schat, 2009; Gia Phan et al., 2013). Although CAV is believed to only infect chickens, and cause severe anemia and immunosuppression in young chickens, few studies reported the presence of CAV in human stool samples (Adair 2000; Chu et al., 2012; Phan et al., 2012). However, whether CAV causes human AGE needs to be determined. Our study added further evidences for the presence of CAV in vomiting and stool samples from AGE patients in two schools. Furthermore, co-infection of norovirus with other AGE-related viruses (e.g. sapovirus and rotavirus) can worse the clinical manifestation (Lekana-Douki et al., 2015). The co-infection of norovirus with CAV was previously reported among two Taiwan children suffering from AGE (Tang et al., 2016). In this study, we also detected co-infection of norovirus and CAV in some AGE patients in the School A, which 
may partially explain why higher percentages of vomiting and abdominal pain occurred in the School A.

On the other hand, the significant difference in noroviruspositive rates suggested that there was no epidemiological link between the two AGE outbreaks in spite that they occurred in two adjacent schools during almost same period (Fig. $1 \mathrm{~b}$ and Table 1). It raised the question where the CAV came from and why the CAV strains from both schools were genetically related. Because CAV infects chickens, the most likely source of CAV was contaminated chicken meat. In addition, the two schools are geographically near to each other, and might purchase the contaminated food from the same source, which provides an explanation for why CAVs in both schools are genetically related. Furthermore, because there were almost half the cases unable to be definitively assigned to any known AGE-related viruses (Table 1), we did not exclude the possibility of bacterial pathogens causing the AGE.

In summary, we report two AGE outbreaks in two adjacent schools in Huzhou, China. One outbreak was caused by the recombinant norovirus GII.P16/GII.2, and another one might be associated with CAV. The association of CAV with AGE needs to be further confirmed by large-scale epidemiological investigations.

Acknowledgments. This work was supported by grants from the Natural Science Foundation of Zhejiang Province (LY16H260001), the National Science and Technology Major Project of China (2017ZX10103009-002), the "One Belt One Road" project of Chinese Academy of Sciences (153831KYSB20170043), and the 133 project of Institut Pasteur of Shanghai, CAS.

\section{References}

Adair BM (2000): Immunopathogenesis of chicken anemia virus infection. Dev. Comp. Immunol. 24, 247-255. https://doi. org/10.1016/S0145-305X(99)00076-2

Ao Y, Wang J, Ling H, He Y, Dong X, Wang X, Peng J, Zhang H, Jin M, Duan Z (2017): Norovirus GII.P16/GII.2-Associated Gastroenteritis, China, 2016. Emerg. Infect. Dis. 23, 1172-1175 . https://doi.org/10.3201/eid2307.170034

Chu DK, Poon LL, Chiu SS, Chan KH, Ng EM, Bauer I, Cheung TK, Ng IH, Guan Y, Wang D, Peiris JS (2012): Characterization of a novel gyrovirus in human stool and chicken meat. J. Clin. Virol. 55, 209-213. https://doi.org/10.1016/j. jev.2012.07.001

Gia Phan T, Phung Vo N, Sdiri-Loulizi K, Aouni M, Pothier P, Ambert-Balay K, Deng X, Delwart E (2013): Divergent gyroviruses in the feces of Tunisian children. Virology 446, 346-348. https://doi.org/10.1016/j.virol.2013.08.020

Kojima S, Kageyama T, Fukushi S, Hoshino FB, Shinohara M, Uchida K, Natori K, Takeda N, Katayama K (2002): Ge- nogroup-specific PCR primers for detection of Norwalklike viruses. J. Virol. Methods 100, 107-114. https://doi. org/10.1016/S0166-0934(01)00404-9

Legoff J, Resche-Rigon M, Bouquet J, Robin M, Naccache SN, Mercier-Delarue S, Federman S, Samayoa E, Rousseau C, Piron P, Kapel N, Simon F, Socié G, Chiu CY (2017): The eukaryotic gut virome in hematopoietic stem cell transplantation: new clues in enteric graft-versus-host disease. Nat. Med. 23, 1080-1085. https://doi.org/10.1038/ $\underline{\mathrm{nm} .4380}$

Lekana-Douki SE, Kombila-Koumavor C, Nkoghe D, Drosten C, Drexler JF, Leroy EM (2015): Molecular epidemiology of enteric viruses and genotyping of rotavirus $\mathrm{A}$, adenovirus and astrovirus among children under 5 years old in Gabon. Int. J. Infect. Dis. 34, 90-95. https://doi.org/10.1016/j. ijid.2015.03.009

Li Y, Deng X, Hu F, Wang J, Liu Y, Huang H, Ma J, Zhang J, Zhang F, Zhang C (2018): Metagenomic analysis identified coinfection with human rhinovirus $\mathrm{C}$ and bocavirus 1 in an adult suffering from severe pneumonia. J. Infect. 76, 311-313. https://doi.org/10.1016/j.jinf.2017.10.012

Lu J, Fang L, Sun L, Zeng H, Li Y, Zheng H, Wu S, Yang F, Song T, Lin J, Ke C, Zhang Y, Vinjé J, Li H (2017): Association of GII.P16-GII.2 Recombinant Norovirus Strain with Increased Norovirus Outbreaks, Guangdong, China, 2016. Emerg. Infect. Dis. 23, 1188-1190. https://doi. org/10.3201/eid2307.170333

Patel MM, Hall AJ, Vinje J, Parashar UD (2009): Noroviruses: a comprehensive review. J. Clin. Virol. 44, 1-8. https://doi. org/10.1016/j.jcv.2008.10.009

Phan TG, Li L, O'Ryan MG, Cortes H, Mamani N, Bonkoungou IJ, Wang C, Leutenegger CM, Delwart E (2012): A third gyrovirus species in human faeces. J. Gen. Virol. 93, 1356-1361. https://doi.org/10.1099/vir.0.041731-0

Schat KA (2009): Chicken anemia virus. Curr. Top. Microbiol. Immunol. 331, 151-183. https://doi.org/10.1007/978-3540-70972-5 10

Tang MB CH, Wu WC, Chou YC, Yu CP (2016): First detection of chicken anemia virus and norovirus genotype II in stool of children with acute gastroenteritis. Southeast Asian J. Trop. Med. Public Health 47, 416-423.

Vinjé J, Vennema H, Maunula L, von Bonsdorff $\mathrm{CH}$, Hoehne M, Schreier E, Richards A, Green J, Brown D, Beard SS, Monroe SS, de Bruin E, Svensson L, Koopmans MP (2003): International collaborative study to compare reverse transcriptase PCR assays for detection and genotyping of noroviruses. J. Clin. Microbiol. 41,1423-1433. https:// doi.org/10.1128/JCM.41.4.1423-1433.2003

Wang J, Li Y, He X, Ma J, Hong W, Hu F, Zhao L, Li Q, Zhang J, Zhang C, Zhang F (2019): Gemykibivirus genome in lower respiratory tract of elderly woman with unexplained acute respiratory distress syndrome. Clin. Infect. Dis. 69 861-864. https://doi.org/10.1093/cid/ciz072 